\section{Cahiers de Narratologie}

Analyse et théorie narratives

14 | 2008

Prose d'idées : formes et savoirs

\title{
Approches passionnelles et dialogiques de la narrativité
}

\section{Raphaël Baroni}

\section{(2) OpenEdition}

\section{Journals}

Édition électronique

URL : http://journals.openedition.org/narratologie/579

DOI : 10.4000/narratologie. 579

ISSN : 1765-307X

Éditeur

LIRCES

\section{Référence électronique}

Raphaël Baroni, «Approches passionnelles et dialogiques de la narrativité », Cahiers de Narratologie [En ligne], 14 | 2008, mis en ligne le 06 mars 2008, consulté le 15 novembre 2019. URL : http:// journals.openedition.org/narratologie/579; DOI : 10.4000/narratologie.579

Ce document a été généré automatiquement le 15 novembre 2019.

\section{(c) (i) (9)}

Cahiers de Narratologie - Analyse et théorie narratives est mis à disposition selon les termes de la licence Creative Commons Attribution - Pas d'Utilisation Commerciale - Pas de Modification 4.0 International. 


\title{
Approches passionnelles et dialogiques de la narrativité
}

\author{
Raphaël Baroni
}

Le but de cet article ${ }^{1}$ est d'entreprendre un rapide tour d'horizon des implications, pour la théorie du récit, des nouvelles orientations théoriques observables aujourd'hui dans de nombreux secteurs des sciences humaines en direction d'une analyse dialogique ou interactionnelle des productions discursives, ainsi que de la mise en évidence de l'intrication fondamentale de l'affect et de la cognition. Il sera ainsi question d'examiner plus précisément les rapports qui existent entre passion et narration dans une approche dialogique de la séquentialité narrative, ou de ce que l'on nomme plus généralement une intrigue.

Il nous faut d'emblée préciser en quelques mots l'usage que nous ferons du terme passion, qui se réfère ici à un phénomène affectif d'ordre général ${ }^{2}$, une forme de pathos qui met en lumière la "passivité » du sujet, la dimension affective de son expérience. Non pas que cette "passivité » serait nécessairement opposée à une "activité » dans le sens ou elle exclurait cette dernière : il s'agit bien plutôt de mettre l'accent sur le fait que l'intentionnalité, qui part du sujet et qui est dirigée vers l'objet, est ici inversée de sorte que l'on s'intéresse davantage à la résistance qu'oppose l'objet au « vouloir » et au " pouvoir » du sujet, que l'on se penche sur la manière dont cet objet affecte le sujet de diverses manières. C'est la relation entre l'agir et le pâtir qui fera l'objet d'une attention particulière, et nous montrerons qu'il est difficile de penser l'un indépendamment de l'autre. Dans cette perspective, s'il est question d'action, cette dernière sera envisagée essentiellement comme une forme de réponse du sujet à une forme de questionnement ou de crise qui l'affecte de l'extérieur.

En fait, dans notre approche de la narrativité, les dimensions active et passive, loin de s'opposer, se complètent et s'éclairent mutuellement. Nous verrons notamment que, dans l'interprétation d'un récit, l'activité cognitive anticipatrice (sous forme de pronostic ou de diagnostic), est en quelque sorte activée par la réticence textuelle manifestée dans la « mise en intrigue » des événements, et cette réticence vise à exciter la curiosité de l'interprète ou à produire du suspense, à l'intriguer, à l'impliquer d'une 
manière ou d'une autre dans l'histoire, c'est-à-dire à accentuer la dimension « passionnante » du texte en entretenant une incertitude provisoire. Nous verrons ainsi qu'il est nécessaire de repenser la corrélation entre ces deux faces, active et passive, qui se manifestent au sein de chaque événement, et notamment dans l'activité interprétative d'une fiction. Ainsi que l'affirme Ricœur, l'« esthétique » au sens large a pour thème « l'exploration des manières multiples dont une œuvre, en agissant sur un lecteur, l'affecte. Cet être affecté a ceci de remarquable qu'il combine, dans une expérience d'un type particulier, une passivité et une activité, qui permettent de désigner comme réception du texte l'action même de le lire »(Ricœur 1985 : 303).

Nous voudrions enfin préciser que le terme "événement » ne sera pas pris dans le sens de l'opposition classique entre événement physique et action associée à un agent humain, telle qu'on la trouve thématisée par exemple dans la philosophie analytique (cf. Revaz 1997), ou dans un sens large qui se réfère à toute forme de changement d'état, mais dans le sens précis de « ce qui fait événement » dans la vie, c'est-à-dire ce qui se manifeste par une « saillance » et qui constitue le « racontable» du récit.

La « mode » des émotions en analyse du discours

La récente et spectaculaire réorientation de la sémiotique greimassienne en direction d'une " sémiotique des passions » est symptomatique de ce changement de cap dans les sciences humaines qui a remis les émotions à la mode (cf. Greimas \& Fontanille 1991; Hénault 1994; Sadoulet 1995; Fontanille \& Zilberberg 1998; Fontanille \& Bordron 2000). Un point crucial que soulèvent ces travaux tient à la mise en évidence d'une corrélation fondatrice, à la racine de la schématisation narrative, entre la tension caractérisant l'événement et l'extension engendrant le procès :

[Au niveau] de la transformation discursive, la forme sensible est celle de l'événement, caractérisé par son éclat et sa saillance, et sa conversion intelligible et extensive engendre le procès, souvent défini comme un "entier » quantifiable et divisible en aspects ; inversement, le procès n'est saisissable pour le sujet du sentir que s'il est modulé par l'intensité qui en fait un événement pour l'observateur. La corrélation fondatrice de la schématisation narrative du discours serait donc celleci :

évènement $<=>$ procès

intensité extensité

(Fontanille \& Zilberberg 1998 : 77)

A travers l'affirmation de cette corrélation fondatrice, la question de la tension peut dès lors revenir au cœur d'une réflexion sémiotique sur la narrativité, et cette approche "passionnelle» du récit recoupe en fait les préoccupations de nombreuses autres disciplines connexes.

Du côté de la linguistique, le passage d'une linguistique structurale à une linguistique énonciative s'est également accompagné d'un regain d'intérêt pour l'analyse des marques de l'émotion dans la langue et par la réhabilitation des travaux de Charles Bally et de Roman Jakobson. Jakobson affirmait en effet que la « fonction émotive, patente dans les interjections, colore à quelques degrés tous nos propos, aux niveaux phonique, grammatical et lexical» (1963: 215). L'essor des approches interactionniste et pragmatique a par ailleurs mis en évidence le fait que certains éléments du texte et du contexte fonctionnaient comme des inducteurs d'émotion chez l'interprète (cf. Plantin, Doury \& Traverso 2000). Mais cette réflexion sur la dimension émotionnelle et interactive de l'interaction verbale n'est pas nouvelle et se trouve déjà esquissée dans la fameuse étude de William Labov (1978) qui visait à mettre en évidence les nombreux 
«procédés évaluatifs» dont se servent les locuteurs pour justifier la pertinence de leurs récits :

Dès lors qu'un événement devient plus ou moins commun, qu'il cesse de violer une règle de comportement établie, il perd son caractère mémorable. C'est pourquoi le narrateur, soumis qu'il est à la pression sociale, se sent toujours contraint de bien montrer que les événements vécus par lui étaient vraiment dangereux et inhabituels, ou que la personne dont il parle a réellement enfreint les règles d'une façon grave et digne d'être rapportée. Bref, ce que disent les procédés évaluatifs, c'est [que] c'était tout le contraire du banal, du quotidien, de l'ordinaire. (Labov 1978: 475-476)

L'approche de Labov souligne donc non seulement qu'il est nécessaire de tenir compte de l'articulation du récit avec sa situation énonciative - l'interaction verbale, directe ou différée, au sein de laquelle il se déroule - mais également de la nature " passionnante " de ce qui est narré, qui doit être conforme aux règles conversationnelles portant sur la pertinence du discours. Jean-Paul Bronckart (1996) souligne lui aussi le fait que le statut dialogique de la séquence narrative (même, et surtout, dans le dialogisme différé propre aux textes littéraires) dépend avant tout de la production d'une tension donnant $\mathrm{du}$ « relief » au discours et assurant sa «mise en intrigue » et sa pertinence au niveau de la relation interlocutive. Ce point de vue est repris et développé par Robert Bouchard (1996), qui distingue les textes à "tension externe ", c'est-à-dire ceux qui permettent de résoudre une tension déjà présente dans l'interaction, comme par exemple les notices de montages ou les recettes de cuisine, des textes à «tension interne ", dont les récits de fiction seraient particulièrement représentatifs.

Parallèlement à ces travaux, qui insistent sur la force de la parole, sur son rapport avec un contexte pragmatique et interactif, il faut signaler également le retour de la rhétorique dans le champ de l'analyse de discours car, comme le résume Claudia Caffi, " au fond, la rhétorique tout entière peut être conçue comme un inventaire infini de dispositifs produisant des émotions » (2000: 89). Cette approche du discours qui insiste sur les moyens à disposition de l'orateur lui permettant de produire du pathos trouve d'ailleurs un prolongement spécifiquement narratif dans la notion de catharsis, cette fonction du discours qui avait été définie, dans la Poétique d'Aristote, comme un effet d'épuration des passions constituant la finalité des arts mimétiques. La tradition rhétorique et poétique héritée d'Aristote a été élargie, modernisée et réinscrite au cœur des questions narratologiques par le biais notamment des travaux de Meir Sternberg $(1990 ; 1992)$, qui distingue, parmi les effets poétiques engendrés par le récit, trois modalités principales, liées chacune à différents «modes d'exposition » de l'action: le suspense (qui dépend d'une narration chronologique), la curiosité (qui est produite par une exposition retardée et énigmatique) et la surprise (qui fait surgir soudainement une information qui avait été dissimulée).

Les propositions théoriques de Sternberg ont également donné naissance, dans le cadre des travaux d'orientation cognitiviste, à la «structural-affect theory» qu'ont développé Brewer et Liechtenstein (1982) et qui est venue donner une assise empirique et psychologique à l'analyse stylistique portant sur les modes d'exposition du récit (cf. également Jose \& Brewer 1985; Brewer 1996). Toujours dans le champ des sciences cognitives, et dès 1979, Beaugrande et Colby ont attiré l'attention sur certains aspects formels rendant les récits plus "intéressants", et donc plus "résistants " dans la mémoire des sujets, aspects qui portent essentiellement sur la gestion d'une incertitude provisoire du texte : «Information is often withheld about linkages of states or events, 
either because it is inferrable or in order to maintain uncertainty and interest » (1979: 48). Ce point de vue était également partagé par Van Dijk (1976) qui soulignait que le récit, pour être acceptable, devait décrire un cours d'événement « remarquable » dans lequel l'intention du sujet était contrariée d'une manière ou d'une autre. Plus récemment, Michel Fayol (2000) a montré que le récit repose généralement - dès lors qu'il atteint un certain degré d'élaboration dans le développement ontogénétique du sujet parlant - sur la transgression d'un "script ", c'est-à-dire sur la rupture d'une routine, ce qui ouvre sur un développement actionnel incertain.

Lorsqu'un événement inattendu survient ou qu'un obstacle surgit, le déroulement des faits ne suit pas un décours habituel. Cette situation devient un objet potentiel de narration [...]. Le caractère inhabituel des faits est perçu et construit comme entrainant une réaction et une (ou plusieurs) action(s) du « héros » de l'histoire visant à rétablir la situation initiale ou à la modifier pour s'adapter. (Fayol 2000 : 195)

Du côté des théories de la réception, Hans-Robert Jauss (1979) a pour sa part réactualisé l'étude de la catharsis (en relation avec la poiesis et l'aisthesis) et cette ouverture, enrichie par une perspective psychanalytique, a été poursuivie notamment par les travaux de Michel Picard sur les fonctions ludiques du récit et sur la question de l'immersion dans des univers fictionnels ${ }^{3}$. Cette question de l'immersion a fait par ailleurs l'objet d'analyses récentes dans une perspective plus cognitiviste, notamment dans l'ouvrage que Jean-Marie Schaeffer a consacré à la fiction (1999) ou dans les travaux de Mary-Laure Ryan sur les rapports entre fiction et réalité virtuelle (2004). Dans un entretien publié sur Vox Poetica, Jean-Marie Schaeffer précise d'ailleurs, au sujet des travaux qu'il a consacrés à l'immersion ludique dans les mondes possibles de la fiction, que les fonctions cognitives sont indissociables de la dimension affective : "Ce terme [cognitif] est souvent lié à l'opposition cognitif / affectif. Or, quand je dis "fonction cognitive", c'est une cognition qui est saturée affectivement. Il me semble qu'il n'y a que cette cognition-là qui soit effective dans la vie réelle. Seules les croyances qui sont saturées affectivement guident nos actions ${ }^{4}$. »

Représentation d'actions ou représentation de passions?

Toutes ces perspectives plus ou moins convergentes que nous venons de passer en revue rendent possible une approche renouvelée de la narrativité dans laquelle la dimension passionnelle (ou passionnante) des événements relatés, ainsi que les traits du suspense, de la curiosité ou de la surprise, qui sont intimement liés au phénomène de la «mise en intrigue» et à la tension interne du discours (que nous baptisons: tension narrative) peuvent enfin (ou à nouveau) jouer les premiers rôles. Pourtant, il semble bien que cette dimension passionnelle de la narrativité demeure encore soit partiellement occultée, soit marginalisée, et cela pour des raisons aussi bien idéologiques qu'esthétiques qu'il nous faut brièvement évoquer.

La critique marxiste et l'esthétique moderniste de «l'art pour l'art» ont longtemps rejeté dans les marges les traits passionnels du récit : la tension ou le suspense étaient jugés comme des artifices racoleurs ne pouvant servir qu'une finalité commerciale et connotant nécessairement des œuvres populaires et déclassées. De ce point de vue, le retour de l'intrigue et le brouillage des valeurs dans l'esthétique postmoderne sont probablement aussi fondamentaux dans la possibilité qui est aujourd'hui offerte de remettre en avant la dimension passionnelle de la narrativité, que l'émergence d'une sémiotique des passions, le retour de la rhétorique et le passage à une linguistique énonciative et pragmatique. 
On constate en effet que les avancées réalisées notamment par Barthes (1970: 1973), Genette (1972) ou Grivel (1973), ont été longtemps limitées par l'esthétique dominant le champ artistique de leur époque. Pour Grivel, par exemple, son analyse de l'intérêt romanesque ne pouvait porter que sur un corpus d'œuvres restreint, composé de romans « populaires » qui s'étalaient entre 1870 et 1880, il ne s'agissait donc nullement d'une analyse narratologique ayant visée générale mais bien d'une étude ayant une connotation fortement historique. Barthes, pour sa part, a décrit dans $S / Z$ (1970) et dans Le Plaisir du texte (1973) les codes qui structurent le récit selon une logique irréversible (codes herméneutique et proaïrétique qui s'apparentent aux effets de curiosité et de suspense) mais, en même temps, il a condamné la lecture linéaire comme une perversion (une forme de voyeurisme) et un asservissement à la logique économique du marché des biens symboliques. Il valorisait à l'inverse une esthétique de la relecture : la lecture « étoilée » et entièrement réversible :

La relecture, opération contraire aux habitudes commerciales et idéologiques de notre société qui recommande de «jeter» l'histoire une fois qu'elle a été consommée ("dévorée »), pour que l'on puisse alors passer à une autre histoire, acheter un autre livre, et qui n'est tolérée que chez certaines catégories marginales de lecteurs (les enfants, les vieillards et les professeurs), la relecture est ici proposée d'emblée, car elle seule sauve le texte de la répétition (ceux qui négligent de relire s'obligent à lire partout la même histoire), le multiplie dans son divers et son pluriel : elle le tire hors de la chronologie interne (« ceci se passe avant ou après cela») et retrouve un temps mythique (sans avant ni après); elle conteste la prétention qui voudrait nous faire croire que la première lecture est une lecture première, naïve, phénoménale, qu'on aurait seulement, ensuite, à " expliquer ", à intellectualiser (comme s'il y avait un commencement de la lecture, comme si tout n'était déjà lu : il n'y a pas de première lecture, même si le texte s'emploie à nous en donner l'illusion par quelques opérateurs de suspense, artifices spectaculaires plus que persuasifs) ; elle n'est plus consommation, mais jeu (ce jeu qui est le retour du différent). (Barthes $1970: 20$ )

Il affirmait encore que « ce qui bloque la réversibilité, voilà ce qui limite le pluriel du texte classique. Ces blocages ont des noms : c'est d'une part la vérité et d'autre part l'empirie : ce précisément contre quoi - ou entre quoi - s'établit le texte moderne" (1970 : 33). Si l'analyse d'une œuvre balzacienne permet d'éclairer ces traits passionnels de la narrativité qui orientent le texte de son nœud vers son dénouement, il est donc affirmé que ces "blocages» de la réversibilité et de la pluralité interprétative ${ }^{5}$ ne caractérisent qu'une esthétique classique révolue. Là encore, on ne toucherait donc pas à un trait fondamental de la narrativité.

Bien que la dimension passionnelle ou "tensive» apparaisse aujourd'hui, pour de nombreux auteurs comme un trait fondamental de la narrativité, et bien que les émotions soient revenues à la mode dans de nombreux secteurs des sciences humaines, il nous semble malgré tout que l'on assiste encore à une certaine résistance dans le renouvellement de la définition $\mathrm{du}$ récit, de sa nature et de sa fonction anthropologique. Peut-être cette résistance tient-elle, ainsi que nous l'avons suggéré, à la difficulté de surmonter certains préjugés esthétiques condamnant, dans le domaine des arts, le recours à des effets émotionnels; mais il y a probablement une cause plus profonde et plus théorique à cette situation : le récit conçu, depuis Aristote, comme une imitation d'action (une mimèsis praxeos) semble, par sa nature même, mettre au premier plan la dimension active de l'expérience. La dimension passive apparait alors comme l'apanage d'autres modes d'expression qui ne sont pas a priori narratifs, tels que le lyrisme par exemple, qui s'oppose à l'épopée ou à la tragédie (cf. Rodriguez 2003). Par 
conséquent, c'est bien la logique de l'action (cf. Bremond), avec sa dynamique projective configurant une temporalité, par opposition à la question apparemment statique des états ou des passions, qui a longtemps occupé l'avant-scène de la réflexion de la narratologie structuraliste.

Mais une telle opposition masque le fait que l'activité et la passivité, la cognition et l' émotion, sont des dimensions indissociables au sein des phénomènes. Ainsi que l'affirmait Ricœur dans un entretien avec Greimas autour de la sémiotique des passions : «D'un point de vue phénoménologique on ne peut rencontrer le problème du pâtir que si on a affaire à des êtres "agissants". [...] Si nous n'étions simplement que des êtres mécaniques, si nous n'étions pas les auteurs de nos actions, capables de passer par les modalités du vouloir et du pouvoir, nous ne saurions pas ce que c'est que les passions. C'est à des êtres agissants qu'il arrive ce quelque chose : souffrir ${ }^{6} »$ (Hénault 1994 : 211).

Entreprendre une action, c'est se heurter à une résistance potentielle, c'est prendre le risque d'échouer dans l'actualisation de son intention. Quand ce risque apparaît nul, quand on nage en pleine routine, il n'y a rien à raconter, le monde est absent, il n'y a pas événement, le temps est réduit à une simple répétition, à un éternel retour du "présent-absent». Au contraire, lorsque l'action est menacée dans son accomplissement, lorsqu'elle est contrariée d'une manière ou d'une autre, lorsque l'on est amené à produire des pronostics incertains sur son succès ou son échec, alors l'événement devient sensible et la temporalité s'approfondit, nos pronostics luttent contre un futur écrasant de sa présence. De même, lorsqu'on nage en plein brouillard, lorsqu'on ne parvient plus à distinguer les objets, les êtres ou à saisir le sens de leurs actions, nos diagnostics s'opposent à un présent ou à un passé lourds de mystères. Il se passe quelque chose et cela méritera peut-être d'être raconté.

Prendre en compte la dimension passionnelle du récit revient ainsi à concevoir que la narrativité consiste précisément en la mise en scène de l'indétermination du monde et/ ou du devenir, c'est le lieu où l'action se représente dans son incertitude, le lieu où peuvent être explorées les obscurités du passé, du présent et du futur dans lesquelles s'enracinent nos angoisses et nos espoirs. Mais c'est aussi le lieu où le monde manifeste sa présence, son extériorité, où les actions apparaissent comme la réponse à une "crise ", et les composantes de l'action ainsi que l'identité du sujet peuvent émerger à travers le temps, se détacher $\mathrm{du}$ fond aveuglant de nos routines quotidiennes. Les contours de l'agir se dessinent grâce à l'ombre projetée par ce qui résiste à notre pouvoir et cette ombre se maintient dans la lumière qu'apportent nos schèmes prévisionnels et explicatifs.

Perspectives pour un renouvellement des questions narratologiques

Une narratologie renouvelée par la prise en compte des dimensions passionnelle et interactive du récit pourrait prendre plusieurs directions. Dans le registre des récits factuels (autobiographies, histoires de vie, historiographie, etc.), si l'on se focalise sur la production, il serait possible d'éclairer l'articulation entre l'événement et sa narrativisation, le récit se noue dans l'expérience et se dénoue par la parole ${ }^{7}$. Au fondement de la production narrative factuelle, on pourrait ainsi mettre en évidence une forme de nouement liée à une crise, l'indétermination du monde et/ou du futur trouverait une réponse dans la production discursive. Dans son ouvrage Pourquoi nous racontons-nous des histoires? Bruner insiste précisément sur ce point : 
Concevoir une histoire, c'est le moyen dont nous disposons pour affronter les surprises, les hasards de la condition humaine, mais aussi pour remédier à la prise insuffisante que nous avons sur cette condition. Les histoires font que ce qui était inattendu nous semble moins surprenant, moins inquiétant: elles domestiquent l'inattendu, le rendent un peu plus ordinaire. "Elle est bizarre, cette histoire, mais elle veut dire quelque chose, non ?" : il nous arrive de réagir ainsi, même en lisant le Frankenstein de Mary Shelley. (Bruner 2002 : 79-80)

La narrativité peut être définie fonctionnellement (au niveau anthropologique) comme la conversion d'une tension existentielle en une tension narrative, comme la transfiguration d'un trait passionnel de l'événement vécu en un trait passionnant de l'événement raconté ${ }^{8}$. Mais si «raconter sa souffrance» peut fournir l'occasion de produire un discours passionnant pour autrui, c'est aussi et surtout, ainsi que le suggèrent Maury-Rouan et Vion (1994), un moyen pour soi d'avoir prise sur cette souffrance, de lui donner sens.

Par ailleurs, du côté de l'interprète cette fois, la tension existentielle se distingue de la tension narrative parce que cette dernière est perçue comme contenant la promesse d'une résolution harmonieuse dont la responsabilité incombe à quelqu'un (le narrateur) qui connaît la fin à l'avance et agence les événements dans son discours dans le but d'atteindre cette «fin » et pas une autre. Le dénouement attendu définit la possibilité émergente d'une compréhension, la promesse d'un « prendre ensemble » du segment existentiel formant une totalité signifiante : le récit thématise le monde et sa dynamique est alternativement projective et rétrospective.

Se pose alors la question des conditions de possibilité d'une « intrigue factuelle » qui aurait pour objectif de rester fidèle à l'événement dans lequel elle s'enracine, d'un récit qui, par conséquent, renoncerait à trahir l'événement en le thématisant, en convertissant les personnes en personnages, en écrasant sa temporalité et en surmontant sa "discordance » initiale par la structure " concordante » d'une totalité fermée. Une comparaison entre la crise vécue et la crise racontée mettrait en tension une visée totalisante et compréhensive du discours (qui tente de domestiquer l'événement saillant, de colmater la brèche ouverte par la «crise » ou par l'« intrusion » d'autrui) avec une visée éthique qui pose l'exigence d'une fidélité de la parole au vécu, et qui renonce par conséquent à intégrer ce dernier dans un mouvement rétrospectif visant une explication ou une totalisation de l'histoire.

Il y a ainsi une contradiction entre la visée pragmatique de la production narrative factuelle, en tant que réponse à une " crise » - cette tendance à la construction d'un sens dont parle Bruner - et l'exigence éthique d'une fidélité du discours par rapport à un événement que l'on sait irrécupérable, dont l'origine et les conséquences se perdent à l'horizon, dans lequel nous ne sommes pas seuls impliqués, et au sein duquel nos actions apparaissent comme une forme de réponse à un affect, à une injonction venue de l'extérieur. Le récit qui vise à rester fidèle à sa source devrait ainsi se tenir dans une tension entre le besoin de faire la lumière sur ce qui est arrivé et la nécessité de préserver la part d'ombre où se tient autrui, un Autre qui n'est pas ramené au Même, mais qui manifeste au contraire son altérité et l'extériorité de son point de vue par sa résistance à notre pouvoir. Autrui qui est intriqué dans notre histoire noue avec nous une intrigue à l'infini et il se trouve également au fondement de notre prise de parole, parce qu'il nous requiert par sa présence. Michel Vanni (2004), dans une réflexion qui recherche à réconcilier la pensée de Lévinas avec la praxis, suggère ainsi que le schème du récit serait adéquat pour transcrire le mouvement de la réquisition par autrui qui 
est au cœur de l'éthique. Il affirme ainsi qu'il est possible d'entendre la série temporelle du récit comme un prolongement de l'affect et non comme une structure formelle se retournant sur l'affect pour l'intégrer, et il conclut qu'on peut considérer la crise ou la mise en question provoquée par l'effraction de l'autre, comme une sorte «d'embrayeur de récit ».

Dans le registre fictionnel cette fois, nos propres recherches (Baroni 2007) se présentent comme une tentative de repenser le concept de séquence narrative dans une perspective passionnelle. L'accent est mis sur la tension interne qui structure rythmiquement le récit, qui inscrit les événements dans une totalité d'abord attendue puis (éventuellement) réalisée. Ainsi que le remarque Jean-Paul Bronckart: "S'il est rarement posé comme tel, le statut dialogique de la séquence narrative est néanmoins évident. [...] Cette séquence se caractérise toujours par la mise en intrigue des événements évoqués. Elle dispose ces derniers de manière à créer une tension, puis à la résoudre, et le suspense ainsi établi contribue au maintien de l'attention du destinataire" (1996: 237). La tension narrative est donc ce qui survient lorsque l'interprète d'un récit est encouragé à prévoir et à attendre un dénouement, cette attente étant caractérisée par une anticipation teintée d'incertitude. La tension narrative contribue au phénomène de la mise en intrigue, elle dépend d'une certaine " réticence » du texte à fournir une information que l'interprète souhaiterait connaître d'emblée, et cette tension interne structure et oriente le discours tout en assurant en même temps sa pertinence au niveau de la relation interlocutive. Nous avons dégagé, à la suite des travaux de Sternberg, deux modalités principales de la tension narrative : le suspense et la curiosité. La curiosité se définit comme un effet qui découle d'une représentation énigmatique de la situation narrative, alors que le suspense dépend pour sa part d'une incertitude qui porte sur le devenir d'une action, dont la représentation respecte au moins partiellement la chronologie. Cette approche, qui tient compte à la fois de la structure de l'action prise en charge par le récit, des modes de textualisation de celle-ci et des effets poétiques qu'elle contribue à produire, consiste par conséquent en une tentative de réconcilier la narratologie thématique (type Bremond) avec la narratologie formelle ou modale (type Genette) alors que la tradition les a longtemps opposées.

La logique actionnelle sous-jacente au récit, que les structuralistes concevaient comme l'architecture immanente du texte, se double ainsi d'une logique discursive qui lui est indissociable et qui se développe au cours du processus interprétatif sur la base de la rencontre entre un mode de textualisation spécifique, marqué par une réticence stratégique, et une activité protensive d'anticipation qui s'oppose à cette réticence. Ce qui permet de déterminer les lieux où le texte se noue et se dénoue, ce sont par conséquent les interrogations que produit l'interprète en un point donné du récit et les pronostics et les diagnostics qu'il établit afin de compenser cette relative perte de contrôle dans l'interaction discursive qui est liée à une réticence du texte. Naturellement, les schémas anticipés sont souvent contredits par le texte, qui parvient ainsi à nous surprendre et nous oblige à objectiver nos attentes et à les corriger. La dynamique polarisée du récit, ainsi que l'a clairement exprimé Umberto Eco (1985), met en jeu deux plans de structuration : une structure anticipée et une structure actualisée, qui ne se recoupent pas nécessairement, et c'est de la tension entre ces deux structures que peut émerger le sens du texte. 
Le dispositif narratif ne rythme effectivement le discours et ne tient en haleine l'interprète que dans la mesure où l'attente est vécue dans une passivité relative, dans la conscience d'une incertitude répondant à la réticence textuelle et soulignant le caractère partiellement imprévisible du texte. La réticence textuelle, qui est donnée comme provisoire, produit alors une tension qui confère du relief au discours et qui oriente l'interprétation par l'attente d'un dénouement à venir. C'est cette double dialectique qui apparaît fondamentale dans la mise en intrigue fictionnelle : d'une part celle qui met en jeu, dans l'interprétation, émotion et cognition (ou incertitude et anticipation) et d'autre part celle qui confronte la réponse anticipée de l'interprète avec la réponse effective du texte, dont pourra éventuellement émerger une surprise mémorable venant renouveler les codes fragiles de la communication narrative.

Si l'on compare maintenant les deux registres (cf. Baroni 2007c), la fonction intrigante que l'on peut associer à la dynamique réticente des récits fictionnels pourrait être contrastée de manière productive avec la fonction configurante que l'on attribue à de nombreux récits factuels. Alors que les premiers défigurent provisoirement le sens des événements pour rejouer la comédie de la passion, pour l'épurer et pour déplacer le sens ordinaire du monde de manière créative, les seconds cherchent au contraire (avec les limites éthiques mentionnées plus haut) à surmonter la brèche ouverte par l'événement, à relogifier le caractère insensé de l'expérience. Dans le premier cas, la discordance est une visée esthétique et en elle réside le plaisir du récit et sa valeur cognitive, dans le second, la discordance est une impureté que la narrativité factuelle a pour charge d'éliminer, et si quelque chose résiste néanmoins, cela tient à la conscience que la synthèse parfaite est impossible, ou alors qu'elle serait une violence symbolique faite à autrui qui est intriqué d'une manière ou d'une autre (en tant que personnage, interlocuteur ou destinataire) dans mon histoire.

Il est donc possible, en soulignant le rôle différentiel de la discordance dans des récits appartenant à des genres distincts, de sortir de la confusion entre configuration (essentiellement rétrospective, visant à substituer à la chronologie une logique causale) et mise en intrigue (essentiellement projective, accentuant la temporalité du discours). Ainsi que l'affirme Mink, la configuration tend à gommer le caractère progressif de la lecture, elle tend également à écraser la temporalité de l'événement relaté : « The more one comes to understand the actual relations among a number of events, as expressed in the story or stories to which they belong, the less one needs to remember dates. " (Mink 1969-1970: 555). A l'inverse, notre approche passionnelle de l'intrigue souligne que cette dernière possède, notamment dans le registre fictionnel, un caractère irréductiblement temporel lié à l'actualisation progressive qu'en fait un interprète : sa fonction dialogique est précisément d'établir une tension interne dans l'interprétation, d'accentuer l'attente d'un dénouement et de contribuer ainsi à polariser et à creuser la temporalité du discours en direction du futur, que ce soit par une forme quelconque de « réticence » textuelle nous contraignant à anticiper le dévoilement d'une information provisoirement cachée, ou par l'évocation d'un événement saillant dont le devenir est imprévisible, ce qui lui confère son épaisseur chronologique sensible. 


\section{BIBLIOGRAPHIE}

Bally, Ch. (1965), Le Langage et la vie, Genève, Librairie Droz.

Baroni, R. (2007), La Tension narrative, Paris, Seuil.

Baroni, R. (2007b), « Fidélité de l'autobiographie et arbitraire du signe », Texte, n 41/42, p. 29-49.

Baroni, R. (2007c), « Histoires vécues, fictions, récits factuels », Poétique, n 151, p. 259-276.

Baroni, R. (à paraître), « Tellability », in Handbook of Narratology, J Pier, W. Schmid, W. Schönert, J. Hühn (dir.), Berlin \& New York, Walter de Gruyter

Barthes, R. (1970), S/Z, Paris, Seuil.

Barthes, R. (1973), Le Plaisir du texte, Paris, Seuil.

Beaugrande, R., \& B. Colby (1979), "Narrative Models of Action and Interaction", Cognitive Science, $\mathrm{n}^{\circ}$ 3, p. 43-66.

Bouchard, R. (1995), “Interaction, discours et "tensions"”, in Modèles de l'interaction verbale, D. Véronique \& R. Vion (éd.), Aix-en-Provence, Publications de l'université de Provence, p. 303-317.

Brewer, W. (1996), “The Nature of Narrative Suspense and the Problem of Rereading”, in Suspense. Conceptualizations, Theoretical Analyses, and Empirical Explorations, P. Vorderer, H. Wulff, and M. Friedrichsen (éd.), Mahwah, Lawrence Erlbaum Associates, p. 107-127.

Brewer, W., \& E. Lichtenstein (1982), "Stories Are to Entertain: A Structural-Affect Theory of Stories", Journal of Pragmatics, n 6, p. 473-486.

Bronckart, J.-P. (1996), Activité langagière, textes et discours. Pour un interactionnisme socio-discursif, Lausanne \& Paris, Delachaux \& Niestlé.

Brooks, P. (1984), Reading for the Plot. Design and Intention in Narrative, Cambridge, Harvard University Press.

Bruner, J. (2002), Pourquoi nous racontons-nous des histoires ?, Paris, Retz.

Caffi, C. (2000), "Aspects du calibrage des distances émotives entre rhétorique et psychologie”, in Les Emotions dans les interactions, Ch. Plantin, M. Doury, and V. Traverso (éd.), Lyon, Presses univeritaires de Lyon, p. 89-104.

Eco, U. (1985), Lector in Fabula, Paris, Grasset.

Fayol, M. (2000), « Comprendre et produire des textes écrits: l'exemple du récit » in L'Acquisition du langage, M. Kail \& M. Fayol (ed), Paris, P.U.F., pp. 183-214.

Fontanille, J., \& J.-F. Bordron (éd.) (2000), Sémiotique du discours et tensions rhétoriques, Paris, Larousse.

Fontanille, J., \& C. Zilberberg (1998), Tension et signification, Paris, Mardaga.

Genette, G. (1972), Figure III, Paris, Seuil.

Gervais, B. (1992), “Lecture: tensions et régies”, Poétique, n 89, p. 105-125.

Greimas, A. J., \& J. Fontanille (1991), Sémiotique des passions, Paris, Seuil.

Grivel, C. (1973), Production de l'intérêt romanesque, Paris \& The Hague, Mouton.

Hénault, A. (1994), Le Pouvoir comme passion, Paris, P.U.F. 
Hoeken, H., \& M. Van Vliet (2000), “Suspense, Curiosity, and Surprise: How Discourse Structure Influences the Affective and Cognitive Processing of a Story”, Poetics, ${ }^{\circ}$ 26, p. 277-286.

Iser, W. (1976), L'Acte de lecture. Théorie de l'effet esthétique, Bruxelles, Pierre Mardaga.

Jakobson, R (1963), Essais de linguistique générale, Paris, Editions de Minuit.

Jauss, H. R. (1979), « La Jouissance esthétique. Les expériences fondamentales de la poiesis, de l'aisthesis et de la catharsis ", Poétique, n 39, p. 261-274.

Jose, P. E., \& W. F. Brewer (1984), “Development of Story Liking: Character Identification, Suspense, and Outcome Resolution”, Developmental Psychology, n 20, p. 911-924.

Jouve, V. (1992), L'Effet-personnage dans le roman, Paris, PUF.

Labov, W. (1978), « La transformation du vécu à travers la syntaxe narrative », in Le Parler ordinaire, (éd.), Paris, Gallimard, p. 457-503.

Mandler, J., and N. S. Johnson (1977), « Remembrance of Things Parsed: Story Structure and Recall », Cognitive Psychology, n 9, p. 111-151.

Maury-Rouan, Claire, and Robert Vion (1994), « Raconter sa souffrance: gestion interactive de la tension narrative », in Le Récit oral, Jacques Bres (dir.), Montpellier, Université Paul-Valéry Montpellier III, p. 215-226.

Mink, L. O. (1969-1970), « History and Fiction as Modes of Comprehension », New Literary History, $\mathrm{n}^{\circ} 1$, p. $541-558$.

Perelman, Ch. (1977), L'Empire rhétorique. Rhétorique et argumentation, Paris, Vrin.

Picard, M. (1986), La Lecture comme jeu: essai sur la littérature, Paris, Editions de Minuit.

Plantin, Ch., M. Doury, and V. Traverso (éd.) (2000), Les Emotions dans les interactions, Lyon, Presses universitaires de Lyon.

Revaz, F. (1997), Les Textes d'action, Paris, Librairie Klinksieck.

Rodriguez, A. (2003), Le Pacte lyrique, Bruxelles, Mardaga.

Ricœur, P. (1983), Temps et récit I, Paris, Seuil, coll. Points.

Ryan, M.-L. (éd.) (2004), Narrative across Media. The Languages of Storytelling, Nebraska, University of Nebraska Press.

Sadoulet, P. (1995), « Convocation du devenir, éclat du survenir et tension dramatique », in Le Devenir, J. Fontanille (éd.), Limoges, PULIM, p. 91-113.

Schaeffer, J.-M. (1999), Pourquoi la fiction ?, Paris, Seuil.

Sternberg, M. (1990), « Telling in time (I): Chronology and Narrative Theory », Poetics Today, $\mathrm{n}^{\circ} 11$, p. 901-948.

Sternberg, M. (1992), « Telling in time (II): Chronology, Teleology, Narrativity », Poetics Today, $\mathrm{n}^{\circ}$ 13 , p. 463-541.

Van Dijk, T. (1976), « Philosophy of action and theory of narrative », Poetics, n 5, p. 287-338.

Vanni, M. (1996), «Stimmung et identité narrative », in Autour de la poétique de Paul Ricoeur, Raphaël Célis et Maurice Sierro (éd.), Etudes de Lettres, p. 89-108. 


\section{NOTES}

1. Cet article reprend certains arguments développés dans le texte d'introduction du dossier « Passion et narration » abrité par le site www.vox-poetica.org. On trouve dans ce dossier plusieurs articles en libre accès tournant autour de la même thématique.

2. Il ne s'agit donc en aucun cas d'une réflexion qui se réfèrerait à la conception étroite du terme associée à la passion amoureuse.

3. Voir également les travaux de Vincent Jouve (1992) sur « l'effet-personnage » qui se situent en partie dans le prolongement des travaux de Picard. Voir aussi les travaux de Peter Brooks (1984), qui croise une approche narratologique post-structuraliste avec une théorie des affects narratifs inspirée des travaux de Freud.

4. Extrait d'un entretien mené par Alexandre Prstojevic pour le site Vox-Poetica, URL : http://www.vox-poetica.org/entretiens/schaeffer.htm.

5. Signalons au passage que l'on ne voit pas pourquoi cette orientation de la lecture, qui favorise dans un premier temps l'économie de la progression, serait incompatible avec une relecture visant une « appropriation » interprétative ultérieure de l'œuvre. Sur ces deux régimes complémentaires de la lecture, cf. Gervais (1992).

6. Peut-être faudrait-il cependant, ainsi que nous y invite Michel Vanni dans le prolongement de la philosophie d'Emmanuel Lévinas, inverser ce rapport et affirmer plutôt que ce n'est qu'à des êtres souffrants, à des êtres répondant à un appel qui vient de l'extérieur, de l'Autre, qu'il arrive ce quelque chose : agir...

7. Voir à ce sujet Baroni (2007b).

8. Sur la question de la « racontabilité », voir Baroni (à paraître).

\section{RÉSUMÉS}

Le but de cet article est d'entreprendre un tour d'horizon des approches qui permettent de penser la séquentialité narrative dans sa dimension dialogique, ainsi que l'intrication fondamentale de l'affect et de la cognition dans la praxis et dans sa représentation. Il devient dès lors possible de concevoir la narrativité comme la mise en scène de l'obscurité du monde et/ou du devenir, et le récit comme la mise en forme de l'action dans l'incertitude constitutive de son déploiement temporel. Dans ce contexte, la fonction intrigante que l'on peut associer à la dynamique réticente des récits fictionnels pourrait être contrastée de manière productive avec la fonction configurante que l'on attribue à de nombreux récits factuels. Alors que les premiers défigurent (au moins provisoirement) le sens des événements pour rejouer la comédie de la passion, pour l'épurer ou pour en déplacer le sens ordinaire, les seconds cherchent au contraire (dans des limites imposées par l'éthique) à surmonter la brèche ouverte par l'événement en le dotant d'un sens.

The aim of this paper is to give a global insight of different theories allowing to deal with the dialogical dimensions of narrative sequence, and the fundamental interaction of affect and cognition in praxis and its representation. Therefore, it becomes possible to consider narrativity as the setting of the obscurity of the world and of its future, and narratives as the shaping of action in the inherent uncertainty of its unfolding in time. In such a context, we can contrast the 
plotting function of most fictional narratives (that produce catharsis by means of the reticence of its discourse structures) with the configurational function of numerous factual narratives (that tend to overcome the breach of canonicity by real events to make sense of it).

INDEX

Mots-clés : récit, catharsis, curiosité, suspense, configuration, intrigue, séquence, dialogisme Keywords : plot, sequence, dialogism, narration, interaction, curiosity

\section{AUTEUR}

\section{RAPHAËL BARONI}

Maître d'enseignement et de recherche à l'Ecole de français langue étrangère de l'Université de Lausanne, Raphaël Baroni est l'auteur de La Tension narrative (Paris, Seuil, coll. Poétique, 2007) et de L'EEuvre du temps (Paris, Seuil, coll. Poétique, 2009). Il est rédacteur en chef de la revue A Contrario 